\title{
Minimally invasive urologic surgery is safe during COVID-19: experience from two high-volume centers in Italy
}

\author{
Giovanni Motterle ${ }^{1} \cdot$ Fabrizio Dal Moro ${ }^{2} \cdot$ Nicola Zanovello $^{1} \cdot$ Alessandro Morlacco $^{1}$ - Deris Gianni Boemo ${ }^{3}$. \\ Fabio Zattoni ${ }^{2} \mathbb{D} \cdot$ Filiberto Zattoni $^{2}$
}

Received: 20 May 2020 / Accepted: 26 May 2020 / Published online: 15 June 2020

๑) Springer-Verlag London Ltd., part of Springer Nature 2020

\begin{abstract}
Potential risks of COVID-19 spread during minimally invasive procedures caused several concerns among surgeons, despite the lack of high-level evidence. Urological robotic and laparoscopic surgery is performed in elective setting in almost all occasions, thus allowing adequate planning and stratification. Two high-volume urological centers in Italy performed 77 robotic and laparoscopic surgeries during the "lockdown" period and adopted various strategies to prevent contamination. First of all, all patients were tested negative with nasopharyngeal swab before the surgical intervention. Patients and personnel were provided adequate personal protective equipment and intraoperative strategies to prevent smoke formation and pneumoperitoneum spread were adopted. No patients nor staff members tested positive for COVID-19 during a 15-day follow-up period. In conclusion, minimally invasive urologic surgery can be safely performed during the pandemic period with adequate planning. We believe that renouncing the benefits of it would be counterproductive, especially in a scenario of long-lasting cohabitation with the virus.
\end{abstract}

Keywords COVID-19 · Laparoscopy $\cdot$ Robotics $\cdot$ Urology

The novel coronavirus pandemic (COVID-19) is presently challenging healthcare systems worldwide [1]. Surgical practice is adapting and will likely be changing further in the future since recent models are suggesting a long-term cohabitation with the virus, until effective treatment or vaccine become available [2]. In this scenario, several recent guidelines have been proposed by International Societies regarding surgery during this particular time, with special focus on uro-oncologic surgery [3]. In the daily urological practice, real life-threatening situations that leave no time for adequate surgical planning are rather uncommon. Thus, it is unlikely that any urological surgery, in particular Minimally Invasive Surgery (MIS) would need to be performed in a COVID-19 patient. However, planning and stratification of

Fabio Zattoni

fabiozattoni@gmail.com

1 Urologic Clinic, Department of Surgical and Oncological Sciences, University of Padova, Padua, Italy

2 Urologic Clinic, "Santa Maria della Misericordia" Hospital, University of Udine, Udine, Italy

3 Management Health Services Department, University Hospital of Padova, Padua, Italy interventions are essential to guarantee an adequate urological care, even on an elective basis, without increasing risks for patients and healthcare professionals.

Recently, in this Journal, Zheng et al. [4] discussed the potential risks of virus spread during minimally invasive procedures, providing some general advices to be applied to these settings. However, evidence either in favor or against the use of MIS after the beginning of COVID-19 pandemic is lacking.

Padova Urology Department serves as an academic referral center in Veneto, Italy. In Padua Province, the first case of COVID-19 was reported on February 21st, 2020 and the subsequent exponential growth of new cases led to the lockdown of the entire Country on March 11th, 2020. Similarly, Udine Hospital is an academic center in Friuli Venezia Giulia, another region in the North-East of Italy. Both Hospitals have served as regional COVID-19 centers, thus since the beginning of the COVID-19 spread the Departments had to re-organize their activity to shift some personnel, resources and equipment to the new dedicated COVID wards. However, we felt compelled to continue providing the usual standards of urological care to our large population of patients, while complying to the new regulations. 
Minimally invasive surgery represents a considerable proportion of ordinary daily practice, accounting for more than $50 \%$ of our major surgeries, performed through robotic or laparoscopic approach. Herein, we report about the approximately 2 months of practice during the COVID-19 "lockdown" period. In the time period between March 9th, and May 1st, 2020, 77 MIS were performed, namely 62 robotic and 15 laparoscopic procedures in both centers combined. Each intervention was performed by high-experienced surgeons with only essential personnel in the operating room, with the suspension of unnecessary training and mentoring activities. Personal Protective Equipment (PPE) was used by staff and patients according to Hospital regulations. Patients were tested with nasopharyngeal swab at admission and all the Department Staff was tested on a weekly basis as per the hospital policy.

No patients tested positive before surgery nor any case of COVID-19 amongst those patients was reported by health authorities in the following 14 days of potential incubation. None of the staff members tested positive during the aforementioned period.

Table 1 summarizes characteristics of patients and interventions performed.

Several authors recently provided recommendations regarding the use of MIS during the COVID-19 pandemic that can be summarized in the following points [5, 6]: (1) patient testing; (2) personnel protection; (3) reduction of smoke formation and spread.

Our experience with MIS and COVID-19 began relatively a long time before the publication of the above recommendations and the choices regulating our activities were based on

Table 1 Summary of minimally invasive surgeries performed

\begin{tabular}{ll}
\hline Age, mean (min-max) & $64.6(25-86)$ \\
Sex, $N(\%)$ & $16(20.8)$ \\
Female & $61(79.2)$ \\
Male & \\
ASA score, $N(\%)$ & $63(81.8)$ \\
1 or 2 & $14(18.2)$ \\
3 or 4 & \\
Type of intervention, $N(\%)$ & $33(42.8)$ \\
RARP & $26(33.8)$ \\
RAPN & $10(13.0)$ \\
VLS Nephrectomy/NUT $=$ & $4(5.2)$ \\
VLS Partial nephrectomy: & $4(5.2)$ \\
Other=4 & $6(7.8)$ \\
15-days Complications (Clavien-Dindo $>3), N(\%)$ & 0 \\
COVID-19 Positive patients & 0 \\
COVID-19-positive staff members & \\
\hline
\end{tabular}

RARP Robot-Assisted Radical Prostatectomy, RAPN Robot-Assisted Partial Nephrectomy, VLS VideoLaparoScopic, NUT NephroUreTerctomy good clinical practice principles. It is noteworthy to pointout that the procedures applied in our Department match what the panels of experts recommended.

1. Testing: when considering elective major surgery (the almost totality of urological practice) an appropriate screening of patients is mandatory. Provided the nondeferability of the intervention [3], we believe that extensive testing on patients can represent the most effective strategy for patient screening, to date. It has been reported that the false negative rate of nasopharyngeal test may vary between 15 and $25 \%$; as a result a negative outcome might not be sufficient for decisions on patient management [7]. In addition to the nasopharyngeal swab, at admission, every patient was tested for body temperature and was required to be asymptomatic for 7 days prior to surgery and not into contact with a known COVID-19 case within the last 14 days. In our opinion, this strategy appears to be the most effective into finding asymptomatic COVID-19 cases, that might be at particular risk of surgical complications [8] and for whom surgery might be postponed in most of the times.

2. Personnel protection: this can be achieved through adequate use of PPE and with the reduction to the minimum of the personnel potentially exposed. Adequate use of PPE appears essential and mandatory in every type of contact with the patient. In our institution, PPE was used as prescribed by healthcare authorities and was made available for all OR staff according to the institutional guidelines. Unnecessary access to OR was suspended for all people not directly involved in surgeries, such as medical students and product specialists, while training and mentoring activities were reduced to a minimum. Lastly, periodic testing on every Department staff member has been performed and it is still ongoing.

3. Reduction of smoke formation and spread: this particular aspect is strongly related to the aerosolization of particles that might occur during MIS. Zheng et al. [4] provided a summary of theoretical risks of spreading the virus through the pneumoperitoneum. It is important to highlight, however, that this theory is based on papers published in the 1990s, on different viruses and that to date there is not even anecdotical report of COVID-19 infection through this route. Additionally, it must be noted that filtration of aerosolized particles may be more difficult during open surgery, where smoke spreading is more likely. Despite the lack of evidence, several authors warned against the use of MIS during COVID-19 pandemic $[9,10]$. In all cases, it is doable and relatively easy to adopt strategies to reduce smoke formation and spread in the OR as suggested by ERUS guidelines such as setting electrosurgery units to the lowest possible set- 
tings and careful desufflation of the pneumoperitoneum [6]. To prevent potential cross-contamination between the patient and the operating room, single-use filters can be applied both for the gas insufflation and evacuation. Another option is represented by AirSeal ${ }^{\circledR}$, that allows a filtration with $0.01 \mu$ ULPA filter with also a dedicated Smoke Evacuation Mode. These strategies, anyhow, are generally advisable under all circumstances and not only during this acute phase of COVID-19 pandemic.

In our opinion, the evidences against MIS appear not to be balanced with its proven benefits. In particular, the lower morbidity and short stay in the hospital that characterize MIS appear to be a clear advantage in a period when health care systems are stressed to the limits. Other aspects that should be emphasized are: (1) blood losses are lower in MIS compared to open surgery and this aspect is particularly important in a period where blood supplies are lacking, due to self-isolation and quarantine. (2) postponed MIS may increase the waiting lists with a double effect: a delay in diagnosis and treatment with possible disease progressions and potential legal issues for hospitals and health systems since there is no high level of evidence to justify such a radical change in ordinary practice. Altogether, these benefits could represent the cost-effectiveness of MIS in this time.

We recognize that our observational study does not prove any cause-effect relationship and besides strict nasopharyngeal swab test protocols, we do not have any serological proof of the absence of the contamination. However, in a period of time where many questions still wait for a definitive answer, high-level evidences are lacking and, probably, will take a long time to become available, even observational experiences may provide useful information for the daily practice. Based on our set of 77 elective MIS performed during the initial COVID-19 pandemic in Italy, we are reporting no case of infection in patients or in the staff. Since early on we had already adopted a strategy comparable to the recommendations published later on. This resulted in an effective model to maintain urological care during COVID-19 pandemic, that is particularly interesting when considering the future potentially long-term cohabitation period with the virus. On the contrary, the reduction in elective activities suggested by some authors [3], appears unrealistic and fails to consider the benefits of MIS, some of which are even strategically more important during a crisis time.

Author contributions Conceptualization: GM, FDM, DGB, FZ, FZ. Methodology: GM, AM, NZ, DGB, FZ. Formal analysis and investigation: GM, FZ. Writing-original draft preparation: GM, AM, FZ. Writing-review and editing: FDM, AM, NZ, FZ. Supervision: FDM, FZ.

\section{Compliance with ethical standards}

Conflict of interest Giovanni Motterle, Fabrizio Dal Moro, Nicola Zanovello, Alessandro Morlacco, Deris Gianni Boemo, Fabio Zattoni and Filiberto Zattoni declare that they have no conflict of interest.

Ethical approval All procedures followed were in accordance with the ethical standards of the responsible committee on human experimentation (institutional and national) and with the Helsinki Declaration of 1975 , as revised in 2000 .

Human and animal rights This article does not contain any studies with human or animal subjects performed by any of the authors.'

Informed consent Consent to participate and for publication: informed consent was obtained from all patients for being included in the study.

\section{References}

1. WHO (2020) Novel Coronavirus. https://www.who.int/emergencie s/diseases/novel-coronavirus-2019. Accessed 1 Apr 2020.

2. Kissler SM, Tedijanto C, Goldstein E, Grad YH, Lipsitch M (2020) Projecting the transmission dynamics of SARS$\mathrm{CoV}-2$ through the postpandemic period. Science. https://doi. org/10.1126/science.abb5793

3. Ribal MJ, Cornford P, Briganti A et al (2020) European association of urology guidelines office rapid reaction group: an organisation-wide collaborative effort to adapt the european association of urology guidelines recommendations to the coronavirus disease 2019 era. Eur Urol. https://doi.org/10.1016/j.eururo.2020.04.056

4. Zheng MH, Boni L, Fingerhut A (2020) Minimally invasive surgery and the novel coronavirus outbreak: lessons learned in China and Italy. Ann Surg. https://doi.org/10.1097/SLA.0000000000 003924

5. Novara G, Giannarini G, De Nunzio C, Porpiglia F, Ficarra V (2020) Risk of SARS-CoV-2 diffusion when performing minimally invasive surgery during the COVID-19 pandemic. Eur Urol. https://doi.org/10.1016/j.eururo.2020.04.015

6. Mottrie A. ERUS (EAU Robotic Urology Section) guidelines during COVID-19 emergency. https://uroweb.org/eau-robotic-urolo gy-section-erus-guidelines-during-covid-19-emergency/.

7. Food and Drug Administration (2020) Fact sheet for healthcare providers. New York SARS-CoV-2 real-rime RT-PCR diagnostic panel. Food and Drug Administration, Silver Spring

8. Lei S, Jiang F, Su W, Chen C, Chen J, Mei W, Zhan L-Y, Jia Y, Zhang L, Liu D, Xia Z-Y, Xia Z (2020) Clinical characteristics and outcomes of patients undergoing surgeries during the incubation period of COVID-19 infection. EClinicalMedicine. https:// doi.org/10.1016/j.eclinm.2020.100331

9. Di Saverio S, Khan M, Pata F, Ietto G, De Simone B, Zani E, Carcano G (2020) Laparoscopy at all costs? Not now during COVID19 and not for acute care surgery and emergency colorectal surgery: a practical algorithm from a Hub Tertiary teaching hospital in Northern Lombardy, Italy. J Trauma Acute Care Surg. https:// doi.org/10.1097/TA.0000000000002727

10. Cohen SL, Liu G, Abrao M, Smart N, Heniford T (2020) Perspectives on surgery in the time of COVID-19: safety first. J Minim Invasive Gynecol. https://doi.org/10.1016/j.jmig.2020.04.003

Publisher's Note Springer Nature remains neutral with regard to jurisdictional claims in published maps and institutional affiliations. 